\title{
Estimation of total antioxidant capacity from diet and supplements in US adults
}

\author{
Meng Yang ${ }^{1}$, Sang-Jin Chung ${ }^{2}$, Chin Eun Chung ${ }^{3}$, Dae-Ok Kim ${ }^{4}$, Won O. Song ${ }^{5}$, Sung I. Koo ${ }^{1}$ \\ and Ock K. Chun ${ }^{1 *}$ \\ ${ }^{1}$ Department of Nutritional Sciences, University of Connecticut, 3624 Horsebarn Road Extension Unit 4017, Storrs, \\ CT 06269, USA \\ ${ }^{2}$ Department of Foods and Nutrition, Kookmin University, Seoul 136-702, South Korea \\ ${ }^{3}$ Department of Food and Nutrition, Ansan College, Ansan 426-701, South Korea \\ ${ }^{4}$ Department of Food Science and Technology, Institute of Life Science and Resources, Kyung Hee University, \\ Yongin 446-701, South Korea \\ ${ }^{5}$ Department of Food Science and Human Nutrition, Michigan State University, East Lansing, MI 48824, USA \\ (Received 18 August 2010 - Revised 9 December 2010 - Accepted 20 December 2010 - First published online 15 February 2011)
}

\begin{abstract}
Given the importance of dietary antioxidants in reducing the risks of chronic diseases, the present study aimed to estimate the intake of total antioxidant capacity (TAC) from diet and dietary supplements of US adults. We utilised the US Department of Agriculture flavonoid and proanthocyanidin databases, dietary supplement data and food consumption data of 4391 US adults aged $19+$ years in the National Health and Nutrition Examination Survey 2001-2. In order to convert the intake data of individual antioxidant compounds to TAC values, the vitamin $\mathrm{C}$ equivalent (VCE) of forty-three antioxidant nutrients measured previously was also applied. Daily TAC averaged $503 \cdot 3$ mg $\mathrm{VCE} / \mathrm{d}$ (approximately $75 \%$ from diet and 25\% from supplements). The energy-adjusted daily TAC level from diet and supplements was higher in women (except for carotenoids), older adults, Caucasian (except for carotenoids), non-alcohol consumers (for vitamin E and proanthocyanidins), subjects with higher income (except for carotenoids) and higher exercise levels than their counterparts $(P<0 \cdot 05)$. TAC was positively associated with daily consumption of fruits and fruit juices, vegetables and vegetable products, beverages, wines and teas $(P<0 \cdot 001)$. Teas, dietary supplements, and fruits and fruit juices were the major sources of dietary TAC of the US population (28, 25 and $17 \%$, respectively), while the contribution of vegetables and vegetable products to TAC was minimal $(<2 \%)$. The present study indicates that antioxidant intake from various diet and supplements contributes to TAC status. TAC levels are different in sociodemographic subgroups of the US population. The relationship between TAC intake and risks of chronic disease warrants further investigation.
\end{abstract}

\section{Key words: Antioxidants: Vitamin C equivalents: Diet recall: Sociodemographic subgroups}

Consumption of fruits and vegetables has been associated with a low incidence and mortality rate of various degenerative diseases including $\mathrm{CVD}^{(1-3)}$ and cancer ${ }^{(4,5)}$. It is not known which dietary constituents are responsible for this association, but it is often assumed that antioxidants play a significant role in this respect. Plant foods contain a variety of compounds with antioxidant activity, including ascorbic acid, tocopherols, carotenoids and phytochemicals such as flavonoids and procyanidins. Since any single antioxidant may not reflect the total antioxidant power of food, the concept of total antioxidant capacity (TAC) has been introduced $^{(6)}$. TAC considers the cumulative/synergistic and protective activities of all the antioxidants present in food or body fluids, thus providing an integrated parameter rather than the simple sum of measurable antioxidants.

Recently, the applicability and scientific appropriateness of the TAC concept have been debated due to the fact that plasma TAC may be affected by plasma protein, uric acid and antioxidant enzymes rather than by antioxidant nutrients and their metabolites directly originating from diets ${ }^{(7)}$. Also, dietary TAC does not reflect bioavailability as determined by absorption and excretion. Furthermore, the successful application of this tool is highly dependent on the completeness and validity of dietary intake data as well as on the accuracy

Abbreviations: ABTS, 2,2'-azino-bis-3-ethylbenzthiazoline-6-sulphonic acid; DR, diet recall; NHANES, National Health and Nutrition Examination Survey; ORAC, oxygen radical absorbance capacity; PA, proanthocyanidin; TAC, total antioxidant capacity; USDA, US Department of Agriculture; VCE, vitamin C equivalents.

*Corresponding author: O. K. Chun, fax +1 860486 3674, email ock.chun@uconn.edu 
of food composition data. Nevertheless, dietary TAC still has a great potential for clinical and public health applications since it exclusively provides the sum of protective activities of dietary antioxidants.

Our research group has recently estimated antioxidant intakes of the US population from diet and dietary supplements by creatively utilising the US Department of Agriculture (USDA) flavonoid databases, food consumption data and dietary supplement data in the National Health and Nutrition Examination Survey (NHANES) 1999-2002 ${ }^{(8-10)}$. To expand our knowledge on the contribution of diets to TAC, a dietary TAC database of the US population has been developed and validated for future application in human antioxidant research $^{(11)}$. However, there is no documentation on the assessment of dietary TAC of a free-living US population due to the limited information of valid antioxidant intake data and measured TAC levels of diverse food items ${ }^{(11)}$. Therefore, the present study aimed to provide the baseline dietary TAC estimation of US adults and sociodemographic subgroups as a premise in order to build the foundation for further investigation of disease prevention and health improvement.

\section{Participants and methods}

\section{Study population}

Individuals aged 19 years and older among the NHANES 2001-2 participants ${ }^{(12)}$ and having reliable and complete diet recall $(\mathrm{DR})^{(13)}$ data as coded by the National Center for Health Statistics ${ }^{(14)}$ were included in the present study ( $n$ 4391). Participants ( $n$ 698) with unreliable and incomplete DR were excluded from the study. There were no significant differences in major outcome variables between included and excluded participants. The NHANES has been conducted by the National Center for Health Statistics to obtain nationally representative information on the health and nutritional status of the US population since the 1970s. All interviewed persons were invited to the mobile examination centre, where the $24 \mathrm{~h}$ DR (midnight to midnight) and questionnaires on dietary supplement use were administered. Written informed consent was obtained from all participants or proxies, and the survey protocol was approved by the Research Ethics Review Board of the National Center for Health Statistics.

\section{Description of datasets}

Details of the datasets used in the present study have been reported in our recent publication ${ }^{(10)}$. Briefly, we created one flavonoid database from two different datasets released in recent years: the USDA database for the flavonoid content of selected foods (2007 update) ${ }^{(15)}$ and the USDA-Iowa State University database on the isoflavone content of foods (2008 update) ${ }^{(16)}$. The combined flavonoid database consisted of twenty-four flavonoid compounds: flavonols (quercetin, kaempferol, myricetin and isorhamnetin), flavones (luteolin and apigenin), flavanones (eriodictyol, hesperetin and naringenin), flavan-3-ols (catechin, epicatechin, theaflavin and thearubigin), anthocyanidins (cyanidin, delphinidin, malvidin, pelargonidin, peonidin and petunidin) and isoflavones (daidzein, genistein, glycitein, biochanin A and formononetin). In order to improve the coverage of the estimated flavonoid intake, we expanded the flavonoid database according to the pre-established protocol that has been described extensively in a separate publication ${ }^{(10)}$. The USDA proanthocyanidin (PA) database ${ }^{(17)}$ released in 2004 complements the USDA flavonoid and isoflavone databases. It contains analytical data generated by the Arkansas Children's Nutrition Center as well as other published analytical data. The PA database includes the food composition data of 205 selected food items for the following PA: monomers, dimers, trimers, 4-6 mers (tetramers, pentamers and hexamers), 7-10 mers (heptamers, octamers, nonamers and decamers) and polymers (degree of polymerisation $>10$ ).

\section{Estimation of antioxidant intakes from diet}

The calculation of dietary antioxidant intake has been described in detail in our preliminary study ${ }^{(10)}$. In summary, we matched the NHANES food consumption data with the USDA flavonoid database following the same procedure: (1) conversion of food items in NHANES dietary recalls to USDA standard reference codes using the food recipe book and food description data file for NHANES food codes; (2) weight adjustment using moisture content; (3) code modification using the USDA food unit conversion search program; (4) linking food intake data with the flavonoid database. Daily individual flavonoid intake from selected foods was determined by multiplying the content of individual flavonoids (mg aglycone equivalents/100 $\mathrm{g}$ food) by the daily consumption $(\mathrm{g} / \mathrm{d})$ of the selected food item. Estimated total intake of individual flavonoids was the sum of individual flavonoid intakes from all food sources reported in the $24 \mathrm{~h}$ DR. Total flavonoid intake was determined by the summation of the total intake of individual flavonoids. Data on individual participant's daily dietary intakes of antioxidant vitamins are available in the NHANES 2001-2 $2^{(12,18)}$.

\section{Estimation of antioxidant intakes from supplements}

A dietary supplement is defined by the Dietary Supplement Health and Education Act of 1994 as 'a product (other than tobacco) intended to supplement the diet that bears or contains one or more of the following dietary ingredients: a vitamin, a mineral, an herb or other botanical, an amino acid or a dietary substance for use by man to supplement the diet by increasing the dietary intake, or a concentrate, metabolite, constituent, extract, or combination of the above ingredients'. The data on dietary supplement use in the NHANES 2001-2 enable investigators to estimate the individual vitamin and mineral intake from dietary supplement use. They provided information about the participants' dietary supplement usage, including supplement counts, supplement records, supplement information, ingredient information and blend information ${ }^{(12)}$. To calculate the intakes of antioxidant nutrients from the supplement, vitamin C, vitamin E, carotenes, Se and flavonoids were selected from the ingredient information 
file. Next, the nutrient composition table of supplements containing the antioxidants was made using the supplement information file. The antioxidant intakes from the supplements were calculated using the supplement counts file, supplement records file and the nutrient composition table of supplements. Participants in the NHANES 2001-2 were questioned specifically about their use of vitamin and mineral supplements. Even though the NHANES dietary supplement data provides comprehensive information on nutrient intake status of the US population from various dietary supplements, limited information is available on flavonoid composition in those products. Furthermore, flavonoid intake from supplements was reported to be less than $2 \%$ in US adults $^{(6)}$. Therefore, flavonoid intake from supplements was not included in the present study. Consequently, the present study includes carotenoids, vitamin $\mathrm{C}$ and vitamin $\mathrm{E}$ from supplements to estimate the total antioxidant intakes.

\section{Analyses of antioxidant capacity of antioxidants}

Antioxidant power of individual antioxidant nutrients expressed as vitamin $\mathrm{C}$ equivalents (VCE) measured by the 2,2'-azino-bis-3-ethylbenzthiazoline-6-sulphonic acid (ABTS) assay has been documented in our previous study ${ }^{(11)}$. Concisely, antioxidant capacities of forty-three major antioxidant nutrients were measured by the ABTS assay conducted according to Kim et al. ${ }^{(19,20)}$. These antioxidants include thirty flavonoids (isorhamnetin, eriodictyol, theaflavin, theaflavin 3-gallate, theaflavin $3^{\prime}$-gallate, theaflavin 3,3'-digallate, petunidin, glycitein, quercetin, kaempferol, myricetin, luteolin, apigenin, hesperetin, naringenin, $(+)$-catechin, $(+)$-gallocatechin, (-)-epicatechin, (-)-epigallocatechin, (-)-epicatechin 3-gallate, (-)-epigallocatechin 3-gallate, cyanidin, delphinidin, malvidin, pelargonidin, peonidin, daidzein, genistein, biochanin A and formononetin), four PA (dimers and trimers), six carotenoids $(\alpha$-carotene, $\beta$-carotene, $\beta$-cryptoxanthin, lutein, lycopene and zeaxanthin), vitamin $\mathrm{E}$ ( $\alpha$-tocopherol and $\gamma$-tocopherol) and vitamin $\mathrm{C}$.

\section{Estimation of total antioxidant capacity from diet and supplements}

Individual antioxidant intake from diet and supplements was determined by multiplying the content of the individual antioxidants (flavonoids, PA, carotenoids, vitamin C and vitamin E) by the daily consumption of each selected food item. The sum of the individual antioxidant intakes was then calculated by summarising individual antioxidant levels from all food sources reported by $24 \mathrm{~h}$ DR and dietary supplement use ${ }^{(21)}$. Antioxidant capacity of each antioxidant consumed daily was calculated by multiplying the consumption data of each antioxidant by its respective antioxidant capacity. TAC from diet and dietary supplements was assessed by summing individual antioxidant capacity and then adjusted by daily total intake and energy intake, which was adjusted to TAC per $4184 \mathrm{~kJ}$ ( $1000 \mathrm{kcal})$. The US adults were subgrouped by sociodemographic and lifestyle variables: age (19-30, 31-50, 51-70 and $70+$ years); sex; ethnicity (non-Hispanic white,
non-Hispanic black, Mexican-American, others); BMI $(\leq 20$, $20-25, \quad 25-30$ and $\left.>30 \mathrm{~kg} / \mathrm{m}^{2}\right)$; poverty income ratios $(<1.85$, $\geq 1.85)$; alcohol consumption (yes or no to 'at least twelve drinks/year'); current smoking (yes or no to 'current smoking' and 'smoked cigarettes, cigars or pipes and/or used chewing tobacco or snuff at least once during the past 30 d'); exercise levels (0, T1, T2 and T3). The exercise levels were expressed as the metabolic equivalent score calculated by combining the intensity level of the leisure-time activities reported, mean duration and frequency.

\section{Statistical analyses}

All statistical analyses were carried out with SAS software, release 8.1, 2000 (SAS Institute, Cary, NC, USA) and the Survey Data Analysis for multi-stage sample designs professional software package (SUDAAN, release 8.0.2, 2003; Research Triangle Institute, Raleigh, NC, USA). Sample weights were applied to all analyses to account for the unequal probability of selection, non-coverage and non-response bias resulting from oversampling of low-income persons, adolescents, the elderly, African-Americans and Mexican-Americans.

The variables on individual and total antioxidant intakes were not normally distributed and they were not normally distributed even after any transformation. However, nonparametric methods could not be used in the analyses since SUDAAN does not work for those methods. Arithmetic means of dietary TAC intake from the daily diet and supplements of subpopulations grouped by sociodemographic and lifestyle variables were determined. Standard error of the means was calculated by the linearisation (Taylor series) variance estimation method for population parameters by SUDAAN. Student's $t$ test and ANOVA were used to compare means for interval scale variables and to test overall differences of TAC intakes by sociodemographic and lifestyle variables such as sex, income, smoking, etc. The trends of TAC intakes by weight of specific food groups consumed were tested using linear contrasts after adjustment for sex, age, ethnicity and total energy intake. The $\chi^{2}$ test was applied for assessing the distributions of categorical variables. Multivariate linear regression analyses were performed to determine the extent to which energy-adjusted TAC intake was explained by dietary behaviours and other sociodemographic factors. The contribution of each food group to the daily TAC was calculated as the ratio of the antioxidant intake from that food group to the total intake from all foods. Values in the tables are presented as means with their standard errors.

\section{Results}

\section{Daily total antioxidant capacity intake from diet and supplements}

Individual antioxidant capacities from diet, supplements and TAC are shown in Table 1. Daily TAC level averages $503.3 \mathrm{mg} \mathrm{VCE} / \mathrm{d}$, approximately $75 \%$ from diet and $25 \%$ from supplements. Vitamin $\mathrm{C}$ and flavonoids are the top two sources contributing to TAC (41 and 39\%, respectively). 
When total energy was adjusted, daily individual antioxidant capacity levels from diet and supplements increased with age in both men and women $(P$ for trend $<0.05$; except for carotenoids in men), with income ( $P$ for trend $<0.05$; except for carotenoids) and exercise level ( $P$ for trend $<0 \cdot 05)$. Energy-adjusted individual antioxidant capacity was higher in women $(P<0.05$; except for carotenoids $)$ and in Caucasians $(P<0.05$; except for carotenoids $)$ than in their counterparts. Alcohol consumers had higher TAC levels from PA than non-consumers $(P<0.05)$. In addition, energyadjusted TAC from diet and supplements were higher in women $(P<0 \cdot 001)$, older adults $(P<0 \cdot 001)$, Caucasians $(P<0.001)$ and those with higher income level $(P<0 \cdot 001)$ and higher exercise level than in their counterparts $(P<0 \cdot 001)$. Alcohol consumption and smoking did not seem to be related to TAC levels. BMI had a weaker association with TAC $(P=0 \cdot 100)$ compared with the sociodemographic factors.

\section{Estimated total antioxidant capacity by food group} consumption

The consumption of specific food or food groups in relation to daily TAC was investigated by testing TAC levels in nonconsumers and tertiles of consumers by major food groups (Table 2). After adjusting for sex, age, ethnicity and total energy intake, TAC levels were positively associated with the daily consumption of fruits and fruit juices, vegetables and vegetable products, beverages, wines and teas $(P<0 \cdot 001)$, whereas bread and grain foods were not related to TAC levels $(P=0 \cdot 724)$.

\section{Major total antioxidant capacity sources consumed in US adults}

Teas, dietary supplements, fruits and fruit juices, and wines were the major food or food groups of TAC based on the $24 \mathrm{~h}$ DR (28, 25, 17 and 5\%, respectively), while vegetables and vegetable products only account for less than $2 \%$. Fig. 1 elucidates the percentage of the major sources contributing to TAC and the corresponding TAC values. The food list of the major TAC sources is presented in Table 3.

\section{Discussion}

Antioxidants found in fruits and vegetables have been assumed to be responsible for the inverse association between higher consumption of these foods and lower risks of chronic diseases $^{(22)}$, while singly administered antioxidant interventions failed to support the promising causal relationship ${ }^{(23-26)}$. Instead of exploring the 'quenching' power of single antioxidants, recently, the concept of TAC has been introduced to express the total synergistic potential of antioxidants for investigating the health effects in food ${ }^{(6)}$. TAC from diet was found to be inversely and independently related to the plasma concentration of high-sensitive C-reactive protein concentration in Italian adults ${ }^{(27)}$, and positively associated with adiponenctin levels by a Greek team ${ }^{(28)}$. Also, it was suggested to be potentially an early estimate of the risk of metabolic syndrome features in Spanish people ${ }^{(29)}$.

A few analytical methods have been developed to measure the synergistic potential of individual food items, differing for scavenging various free radicals and for measuring different endpoints $^{(30)}$. The commonly used methods include Trolox equivalent antioxidant capacity ${ }^{(31)}$, oxygen radical absorbance capacity (ORAC) $)^{(32)}$, total radical-trapping antioxidant parameters $^{(33)}$, ferric-reducing antioxidant power ${ }^{(34)}$, 1,1-diphenyl-2-picrylhydrazyl ${ }^{(35)}$ and ABTS $^{(36)}$ assays ${ }^{(37)}$. Among these methods, the ABTS assay developed by Kim et al. ${ }^{(38)}$ and expressed in VCE antioxidant capacity was used in the present study to estimate TAC. The ABTS (VCE antioxidant capacity) assay utilises quantitative concepts in reference to the familiar vitamin $\mathrm{C}$ to measure both hydrophilic and lipophilic antioxidant activities, and its weight-based expression enables researchers to link weight-based food consumption data to estimate $\mathrm{TAC}^{(38)}$.

$\mathrm{DR}^{(39)}$ and $\mathrm{FFQ}^{(30,40)}$ have been commonly used to assess dietary TAC by summation of known TAC values of different food items measured by total radical-trapping antioxidant parameters or ORAC, which, to large extent, depends on the ORAC or total radical-trapping antioxidant parameter food datasets limited to the species and amounts of fruits and vegetables. Although, in 2007, the USDA released an ORAC dataset of 277 selected food items based on a meta-analysis ${ }^{(41)}$, it is unlikely to be used in different countries because of various food availability and nutrient fortification laws. Since it is impractical to measure the TAC of every food that each individual person consumes, we creatively estimated the dietary TAC theoretically by summation of antioxidant capacities of individual antioxidants consumed/d. This theoretical TAC of foods has been proven to be positively correlated with the different TAC values determined analytically by ABTS and 1,1-diphenyl-2-picrylhydrazyl assays and with TAC values from matched forty-four food items from the USDA ORAC database ${ }^{(11)}$. To our best knowledge, this is the first time such a method has been used to estimate dietary TAC of US adults. This approach to calculate dietary TAC would not be limited by specific food items, whose antioxidant capacity has to be measured in advance.

Daily TAC of $503.3 \mathrm{mg}$ VCE in the present study is lower than what we reported previously in 2005 based on experimental data from fruits and vegetables purchased locally in the New York area $(591 \mathrm{mg} \text { VCE })^{(21)}$. In the present study, antioxidants from both diet and supplements played pivotal roles in the daily TAC of US adults. Individual antioxidant contributions to TAC were in the following order: vitamin $\mathrm{C}>$ flavonoids $>$ proanthocyanins $>$ vitamin $\quad \mathrm{E}>$ carotenoids. TAC from supplements took account for almost one-fourth of TAC, and particularly more than half of vitamin C contribution to TAC was from supplements, indicating that supplementation is a major source for vitamin $C$ intake in US adults. These results were in accordance with the previous report that about $48 \%$ of US adults take at least one supplement/d and that vitamin C intake from supplements are higher than that from diet ${ }^{(10)}$. Dietary phenolic 
Table 1. Total antioxidant capacity (TAC) from diet and supplements of US adults aged $19+$ years and its subgroups by sociodemographic and lifestyle factors in the National Health and Nutrition Examination Survey 2001-2

(Mean values with their standard errors)

\begin{tabular}{|c|c|c|c|c|c|c|c|c|c|c|c|c|c|}
\hline & \multirow{3}{*}{$\begin{array}{l}\text { Stratified } \\
\text { sample }(n)\end{array}$} & \multicolumn{6}{|c|}{ Vitamin C† (mg VCE/d) } & \multicolumn{6}{|c|}{ Vitamin E (mg VCE/d) } \\
\hline & & \multicolumn{2}{|c|}{ Diet } & \multicolumn{2}{|c|}{ Suppl } & \multicolumn{2}{|c|}{ Total } & \multicolumn{2}{|c|}{ Diet } & \multicolumn{2}{|c|}{ Suppl } & \multicolumn{2}{|c|}{ Total } \\
\hline & & Mean & SE & Mean & SE & Mean & SE & Mean & SE & Mean & SE & Mean & SE \\
\hline \multicolumn{14}{|l|}{ Sex } \\
\hline Men & 2247 & $103 \cdot 2$ & 4.8 & $96 \cdot 1$ & 9.7 & $199 \cdot 3^{\star \star \star}$ & 11.4 & $2 \cdot 2$ & 0.1 & $10 \cdot 5$ & $1 \cdot 0$ & $12 \cdot 8^{\star \star \star}$ & 1.0 \\
\hline Women & 2144 & $84 \cdot 2$ & $2 \cdot 2$ & $125 \cdot 1$ & $12 \cdot 6$ & $209 \cdot 3$ & 12.5 & $1 \cdot 7$ & 0.1 & $15 \cdot 3$ & $1 \cdot 1$ & $17 \cdot 1$ & $1 \cdot 1$ \\
\hline \multicolumn{14}{|l|}{ Age (years) } \\
\hline $19-30$ & 946 & $102 \cdot 1$ & $6 \cdot 2$ & $49 \cdot 1$ & 5.8 & $151 \cdot 2^{\star \star \star}$ & $10 \cdot 0$ & $2 \cdot 0$ & 0.0 & 3.4 & 0.5 & $5 \cdot 4^{\star \star *}$ & 0.5 \\
\hline $31-50$ & 1466 & 89.5 & $5 \cdot 5$ & $97 \cdot 7$ & $12 \cdot 8$ & $187 \cdot 2$ & $12 \cdot 8$ & $2 \cdot 0$ & 0.1 & $9 \cdot 2$ & $1 \cdot 1$ & $11 \cdot 2$ & 1.0 \\
\hline $51-70$ & 1228 & $96 \cdot 6$ & $4 \cdot 1$ & $164 \cdot 2$ & $20 \cdot 9$ & $260 \cdot 8$ & 21.6 & $2 \cdot 0$ & 0.1 & $22 \cdot 3$ & $2 \cdot 0$ & $24 \cdot 3$ & $2 \cdot 0$ \\
\hline $70+$ & 751 & $96 \cdot 6$ & 3.6 & $147 \cdot 7$ & 14.5 & $233 \cdot 8$ & $14 \cdot 6$ & 1.6 & 0.1 & $23 \cdot 1$ & $1 \cdot 7$ & $24 \cdot 8$ & 1.7 \\
\hline \multicolumn{14}{|l|}{ Ethnicity } \\
\hline $\begin{array}{l}\text { Non-Hispanic } \\
\text { white }\end{array}$ & 2302 & $89 \cdot 3$ & 3.5 & $131 \cdot 5$ & $12 \cdot 4$ & $220 \cdot 8^{\star \star}$ & $13 \cdot 1$ & $2 \cdot 0$ & 0.1 & $15 \cdot 4$ & $1 \cdot 1$ & $17 \cdot 4^{\star \star \star}$ & $1 \cdot 1$ \\
\hline $\begin{array}{l}\text { Non-Hispanic } \\
\text { black }\end{array}$ & 849 & $100 \cdot 8$ & $6 \cdot 9$ & $40 \cdot 8$ & $5 \cdot 9$ & $141 \cdot 6$ & 8.5 & $1 \cdot 8$ & $0 \cdot 1$ & $6 \cdot 5$ & $1 \cdot 0$ & $8 \cdot 3$ & $1 \cdot 0$ \\
\hline $\begin{array}{l}\text { Mexican- } \\
\text { American }\end{array}$ & 942 & $114 \cdot 8$ & $3 \cdot 8$ & $45 \cdot 3$ & $7 \cdot 7$ & $160 \cdot 1$ & $7 \cdot 8$ & 1.9 & 0.0 & $4 \cdot 8$ & 0.7 & $6 \cdot 6$ & 0.7 \\
\hline Others & 298 & 104.4 & $6 \cdot 4$ & 67.5 & $13 \cdot 2$ & 171.9 & $16 \cdot 3$ & $1 \cdot 7$ & 0.1 & $6 \cdot 3$ & $1 \cdot 3$ & $8 \cdot 1$ & 1.3 \\
\hline \multicolumn{14}{|l|}{ BMI $\left(\mathrm{kg} / \mathrm{m}^{2}\right)$} \\
\hline$\leq 20$ & 248 & $93 \cdot 6$ & 8.5 & 85.6 & $25 \cdot 3$ & $179 \cdot 2$ & 27.9 & 1.9 & 0.1 & 11.4 & 3.7 & $13 \cdot 3$ & $3 \cdot 7$ \\
\hline 20 to $\leq 25$ & 1181 & $105 \cdot 2$ & $4 \cdot 1$ & $127 \cdot 9$ & $20 \cdot 6$ & 233.0 & 21.2 & $2 \cdot 1$ & 0.1 & $14 \cdot 2$ & 1.7 & $16 \cdot 3$ & 1.7 \\
\hline 25 to $\leq 30$ & 1530 & $89 \cdot 2$ & $3 \cdot 3$ & $112 \cdot 3$ & 7.9 & 201.4 & 8.2 & 1.9 & 0.0 & $12 \cdot 5$ & 0.9 & $14 \cdot 4$ & 0.9 \\
\hline$>30$ & 1248 & 88.4 & $4 \cdot 3$ & $100 \cdot 7$ & $11 \cdot 3$ & $189 \cdot 1$ & $13 \cdot 0$ & $2 \cdot 0$ & 0.1 & $12 \cdot 8$ & $1 \cdot 3$ & $14 \cdot 8$ & $1 \cdot 3$ \\
\hline \multicolumn{14}{|l|}{ PIR $\ddagger$} \\
\hline$<1.0$ & 695 & $89 \cdot 0$ & 3.4 & $51 \cdot 6$ & 8.7 & $140 \cdot 6^{\star \star *}$ & 9.8 & $2 \cdot 0$ & 0.1 & $5 \cdot 6$ & $1 \cdot 2$ & $7 \cdot 3^{\star * *}$ & $1 \cdot 2$ \\
\hline $1 \cdot 0-1 \cdot 3$ & 411 & $82 \cdot 2$ & $9 \cdot 7$ & $55 \cdot 8$ & $11 \cdot 0$ & 137.9 & $16 \cdot 7$ & $1 \cdot 7$ & 0.1 & $8 \cdot 3$ & $2 \cdot 1$ & 9.9 & $2 \cdot 1$ \\
\hline $1.3-1.85$ & 530 & 78.5 & $5 \cdot 6$ & 60.6 & $10 \cdot 9$ & $139 \cdot 1$ & $12 \cdot 2$ & $1 \cdot 8$ & 0.1 & $11 \cdot 0$ & $1 \cdot 8$ & $12 \cdot 8$ & 1.8 \\
\hline$\geq 1.85$ & 2467 & 98.9 & 3.4 & 133.8 & $14 \cdot 1$ & 232.7 & 13.9 & $2 \cdot 1$ & 0.1 & $14 \cdot 7$ & $1 \cdot 0$ & $16 \cdot 8$ & 1.0 \\
\hline \multicolumn{14}{|c|}{ Alcohol consumption§ } \\
\hline No & 1231 & 94.8 & 4.4 & 105.9 & 11.4 & $200 \cdot 8$ & $12 \cdot 3$ & 1.9 & 0.1 & $14 \cdot 7$ & 1.5 & $16 \cdot 6^{\star}$ & 1.5 \\
\hline Yes & 2766 & $92 \cdot 3$ & 3.4 & $117 \cdot 1$ & $12 \cdot 9$ & 209.4 & $13 \cdot 0$ & $2 \cdot 0$ & 0.1 & $13 \cdot 0$ & $1 \cdot 1$ & $15 \cdot 0$ & $1 \cdot 1$ \\
\hline \multicolumn{14}{|c|}{ Current smoking\|l| } \\
\hline No & 2085 & $102 \cdot 5$ & $2 \cdot 6$ & $109 \cdot 0$ & $7 \cdot 0$ & 211.5 & 8.4 & $2 \cdot 0$ & 0.1 & $13 \cdot 6$ & $1 \cdot 1$ & $15 \cdot 6$ & $1 \cdot 1$ \\
\hline Yes & 2080 & 83.8 & 4.6 & $116 \cdot 0$ & $15 \cdot 4$ & $199 \cdot 7$ & $15 \cdot 5$ & 1.9 & 0.1 & $12 \cdot 8$ & $1 \cdot 1$ & $14 \cdot 7$ & $1 \cdot 1$ \\
\hline \multicolumn{14}{|l|}{ Exercise levelף } \\
\hline 0 & 1473 & 75.5 & $3 \cdot 8$ & $67 \cdot 0$ & $9 \cdot 0$ & $142 \cdot 4^{\star \star}$ & 9.4 & $1 \cdot 7$ & 0.1 & 9.4 & 0.9 & $11 \cdot 1^{\star *}$ & 0.9 \\
\hline $\mathrm{T} 1$ & 838 & 84.9 & 4.7 & 123.7 & $20 \cdot 4$ & $208 \cdot 6$ & 21.8 & $2 \cdot 0$ & 0.1 & $10 \cdot 8$ & 1.3 & $12 \cdot 8$ & 1.3 \\
\hline T2 & 835 & $107 \cdot 0$ & $4 \cdot 8$ & $127 \cdot 2$ & $16 \cdot 4$ & 234.1 & $16 \cdot 5$ & $2 \cdot 1$ & 0.1 & $16 \cdot 8$ & 1.4 & $18 \cdot 9$ & 1.5 \\
\hline T3 & 886 & $112 \cdot 0$ & $5 \cdot 2$ & $140 \cdot 4$ & $15 \cdot 8$ & 252.5 & $16 \cdot 8$ & $2 \cdot 2$ & 0.1 & $15 \cdot 2$ & $1 \cdot 7$ & $17 \cdot 4$ & 1.6 \\
\hline
\end{tabular}


WS British Journal of Nutrition

Table 1. Continued

\begin{tabular}{|c|c|c|c|c|c|c|c|c|c|c|c|c|c|c|c|c|}
\hline & \multicolumn{6}{|c|}{ Carotenoids (mg VCE/d) } & \multirow{2}{*}{\multicolumn{2}{|c|}{$\begin{array}{c}\begin{array}{c}\text { Flavonoids } \\
\text { (mg VCE/d) }\end{array} \\
\text { Diet }\end{array}$}} & \multirow{2}{*}{\multicolumn{2}{|c|}{$\begin{array}{c}\begin{array}{c}\text { PA } \\
(\mathrm{mg} \mathrm{VCE} / \mathrm{d})\end{array} \\
\text { Diet }\end{array}$}} & \multicolumn{6}{|c|}{ TAC (mg VCE/d) } \\
\hline & \multicolumn{2}{|c|}{ Diet } & \multicolumn{2}{|c|}{ Suppl } & \multicolumn{2}{|c|}{ Total } & & & & & \multicolumn{2}{|c|}{ Diet } & \multicolumn{2}{|c|}{ Suppl } & \multicolumn{2}{|c|}{ Total } \\
\hline & Mean & SE & Mean & SE & Mean & SE & Mean & SE & Mean & SE & Mean & SE & Mean & SE & Mean & SE \\
\hline \multicolumn{17}{|l|}{ Sex } \\
\hline Men & 5.5 & 0.3 & $0 \cdot 1$ & 0.0 & $5 \cdot 6$ & 0.3 & $210 \cdot 7^{* *}$ & $14 \cdot 3$ & $92 \cdot 9^{\star \star}$ & $6 \cdot 9$ & $414 \cdot 6$ & $21 \cdot 0$ & $106 \cdot 7$ & $10 \cdot 3$ & $521 \cdot 3^{\star \star \star}$ & 23.9 \\
\hline Women & 3.9 & 0.2 & 0.1 & 0.0 & 3.9 & 0.2 & $178 \cdot 4$ & $10 \cdot 2$ & $77 \cdot 3$ & 4.5 & $345 \cdot 4$ & $14 \cdot 8$ & 140.5 & $13 \cdot 2$ & 485.9 & $19 \cdot 2$ \\
\hline \multicolumn{17}{|l|}{ Age (years) } \\
\hline $19-30$ & $5 \cdot 3$ & 0.6 & $0 \cdot 1$ & 0.0 & $5 \cdot 3^{\star}$ & 0.6 & $168 \cdot 6^{\star \star \star}$ & $16 \cdot 9$ & $72 \cdot 7^{\star \star}$ & $7 \cdot 5$ & $350 \cdot 6$ & $23 \cdot 8$ & 52.5 & $6 \cdot 1$ & $403 \cdot 2^{\star \star \star}$ & $23 \cdot 0$ \\
\hline $31-50$ & 4.6 & 0.3 & $0 \cdot 1$ & 0.0 & 4.7 & 0.3 & $200 \cdot 4$ & $12 \cdot 3$ & $92 \cdot 2$ & $6 \cdot 3$ & 388.7 & $18 \cdot 2$ & $107 \cdot 0$ & 13.4 & $495 \cdot 7$ & $24 \cdot 2$ \\
\hline $51-70$ & $4 \cdot 8$ & 0.3 & $0 \cdot 1$ & 0.0 & 4.9 & 0.3 & $219 \cdot 1$ & $18 \cdot 2$ & $90 \cdot 7$ & 8.4 & $413 \cdot 2$ & $26 \cdot 6$ & $186 \cdot 6$ & $22 \cdot 0$ & 599.8 & $41 \cdot 0$ \\
\hline $\begin{array}{c}70+ \\
\text { Ethnicity }\end{array}$ & 3.6 & 0.3 & 0.1 & 0.0 & $3 \cdot 7$ & 0.3 & $157 \cdot 8$ & $13 \cdot 8$ & $65 \cdot 3$ & $6 \cdot 3$ & $314 \cdot 4$ & $19 \cdot 2$ & $171 \cdot 0$ & $15 \cdot 2$ & $485 \cdot 4$ & $27 \cdot 1$ \\
\hline $\begin{array}{l}\text { Non-Hispanic } \\
\text { white }\end{array}$ & 4.9 & 0.3 & 0.1 & 0.0 & $5 \cdot 0$ & 0.3 & $213 \cdot 5^{\star \star}$ & $14 \cdot 8$ & $94 \cdot 5^{\star \star \star}$ & $7 \cdot 0$ & $404 \cdot 2$ & 21.9 & $147 \cdot 0$ & $13 \cdot 0$ & $551 \cdot 2^{* * *}$ & $26 \cdot 0$ \\
\hline $\begin{array}{l}\text { Non-Hispanic } \\
\text { black }\end{array}$ & $3 \cdot 8$ & 0.3 & 0.1 & 0.0 & $3 \cdot 8$ & 0.3 & $136 \cdot 2$ & $8 \cdot 0$ & $61 \cdot 3$ & $4 \cdot 0$ & $303 \cdot 9$ & $15 \cdot 7$ & $47 \cdot 3$ & $6 \cdot 7$ & $351 \cdot 2$ & 14.5 \\
\hline $\begin{array}{l}\text { Mexican- } \\
\text { American }\end{array}$ & 4.7 & 0.3 & 0.1 & 0.0 & 4.7 & 0.2 & $128 \cdot 1$ & 5.9 & 49.7 & $2 \cdot 6$ & 299.1 & $7 \cdot 3$ & $50 \cdot 1$ & $8 \cdot 2$ & $349 \cdot 2$ & 11.6 \\
\hline \multicolumn{17}{|l|}{ BMI $\left(\mathrm{kg} / \mathrm{m}^{2}\right)$} \\
\hline$\leq 20$ & $5 \cdot 0$ & 0.9 & $0 \cdot 1$ & 0.0 & $5 \cdot 0$ & 0.9 & $175 \cdot 0$ & $28 \cdot 8$ & $86 \cdot 0$ & $13 \cdot 3$ & 361.5 & $44 \cdot 0$ & $97 \cdot 0$ & $28 \cdot 1$ & $458.5^{\star}$ & $58 \cdot 7$ \\
\hline 20 to $\leq 25$ & 4.8 & 0.4 & 0.1 & 0.0 & 4.9 & 0.4 & $198 \cdot 2$ & $17 \cdot 3$ & $86 \cdot 2$ & $7 \cdot 1$ & 396.4 & 23.9 & $142 \cdot 2$ & $21 \cdot 8$ & $538 \cdot 6$ & $33 \cdot 6$ \\
\hline 25 to $\leq 30$ & 4.6 & 0.3 & $0 \cdot 1$ & 0.0 & 4.7 & 0.3 & 214.7 & $15 \cdot 3$ & $95 \cdot 6$ & $7 \cdot 6$ & $406 \cdot 0$ & 21.5 & $124 \cdot 8$ & 8.7 & $530 \cdot 7$ & $22 \cdot 8$ \\
\hline$>30$ & $4 \cdot 8$ & 0.4 & $0 \cdot 1$ & 0.0 & 4.9 & 0.4 & $176 \cdot 3$ & $13 \cdot 0$ & 73.9 & $5 \cdot 8$ & $345 \cdot 3$ & $16 \cdot 8$ & 113.7 & 11.5 & 459.0 & $15 \cdot 6$ \\
\hline \multicolumn{17}{|l|}{ PIR $\ddagger$} \\
\hline$<1.0$ & 4.6 & 0.4 & 0.1 & 0.0 & 4.6 & 0.4 & $138 \cdot 3^{\star *}$ & $16 \cdot 4$ & $59 \cdot 5^{\star}$ & $5 \cdot 7$ & 293.0 & $19 \cdot 9$ & $57 \cdot 3$ & 8.9 & $350 \cdot 3^{\star \star *}$ & $21 \cdot 7$ \\
\hline $1 \cdot 0-1 \cdot 3$ & $4 \cdot 3$ & 0.6 & 0.1 & 0.0 & 4.4 & 0.6 & $151 \cdot 1$ & $15 \cdot 9$ & $63 \cdot 2$ & $6 \cdot 2$ & 302.5 & 23.0 & $64 \cdot 1$ & $12 \cdot 9$ & 366.5 & $29 \cdot 7$ \\
\hline $1.3-1.85$ & 4.6 & 0.5 & $0 \cdot 1$ & 0.0 & $4 \cdot 7$ & 0.4 & $146 \cdot 7$ & $20 \cdot 6$ & $73 \cdot 8$ & $12 \cdot 6$ & 305.5 & $35 \cdot 2$ & $71 \cdot 7$ & $12 \cdot 1$ & $377 \cdot 2$ & $39 \cdot 1$ \\
\hline$\geq 1.85$ & $4 \cdot 8$ & 0.3 & 0.1 & 0.0 & 4.9 & 0.3 & 214.4 & $13 \cdot 0$ & 93.6 & $6 \cdot 3$ & 413.7 & $19 \cdot 1$ & $148 \cdot 6$ & $14 \cdot 7$ & $562 \cdot 3$ & $21 \cdot 9$ \\
\hline \multicolumn{17}{|c|}{ Alcohol consumption§ } \\
\hline No & 4.5 & 0.5 & $0 \cdot 1$ & 0.0 & 4.6 & 0.5 & $159 \cdot 3$ & $12 \cdot 5$ & $59 \cdot 9^{\star \star}$ & $3 \cdot 3$ & $320 \cdot 4$ & $13 \cdot 0$ & $120 \cdot 8$ & $12 \cdot 0$ & $441 \cdot 1$ & $20 \cdot 0$ \\
\hline Yes & 4.7 & 0.2 & 0.1 & 0.0 & $4 \cdot 8$ & 0.2 & 211.6 & $12 \cdot 9$ & $95 \cdot 8$ & $6 \cdot 5$ & $406 \cdot 4$ & $18 \cdot 8$ & $130 \cdot 2$ & $13 \cdot 7$ & 536.6 & $23 \cdot 3$ \\
\hline \multicolumn{17}{|c|}{ Current smoking $\|$} \\
\hline No & $5 \cdot 0$ & 0.3 & $0 \cdot 1$ & 0.0 & $5 \cdot 1$ & 0.3 & $199 \cdot 8$ & $14 \cdot 2$ & $82 \cdot 7$ & $5 \cdot 8$ & 392.0 & $19 \cdot 8$ & $122 \cdot 7$ & 7.4 & 514.7 & $23 \cdot 3$ \\
\hline Yes & 4.4 & 0.2 & 0.1 & 0.0 & 4.5 & 0.2 & $192 \cdot 2$ & $13 \cdot 8$ & 88.6 & $7 \cdot 0$ & 370.9 & $20 \cdot 9$ & $128 \cdot 8$ & $16 \cdot 1$ & 499.8 & $26 \cdot 7$ \\
\hline \multicolumn{17}{|l|}{ Exercise levelף } \\
\hline 0 & $4 \cdot 1$ & 0.3 & $0 \cdot 1$ & 0.0 & $4 \cdot 2^{*}$ & 0.3 & $169 \cdot 9^{\star \star}$ & $10 \cdot 6$ & $73 \cdot 0^{\star \star}$ & $4 \cdot 2$ & $324 \cdot 2$ & $15 \cdot 0$ & 76.4 & $9 \cdot 6$ & $400 \cdot 5^{\star \star \star}$ & $11 \cdot 6$ \\
\hline T1 & 4.6 & 0.5 & $0 \cdot 1$ & 0.0 & 4.7 & 0.5 & $186 \cdot 2$ & $18 \cdot 7$ & $87 \cdot 0$ & 8.4 & 364.6 & $25 \cdot 6$ & 134.6 & $20 \cdot 6$ & $499 \cdot 2$ & $32 \cdot 6$ \\
\hline T2 & $5 \cdot 2$ & 0.3 & 0.1 & 0.0 & $5 \cdot 3$ & 0.3 & 203.4 & $13 \cdot 3$ & 87.4 & $7 \cdot 1$ & 405.0 & $19 \cdot 6$ & $144 \cdot 1$ & $16 \cdot 9$ & 549.1 & $26 \cdot 1$ \\
\hline T3 & $5 \cdot 1$ & 0.3 & 0.1 & 0.0 & $5 \cdot 2$ & 0.3 & $225 \cdot 7$ & $18 \cdot 9$ & 98.2 & $8 \cdot 3$ & $443 \cdot 3$ & $27 \cdot 7$ & 155.7 & $16 \cdot 9$ & 599.0 & $33 \cdot 2$ \\
\hline
\end{tabular}

VCE, vitamin C equivalents; Suppl, supplements; PA, proanthocyanidins; PIR, poverty income ratio; MET, metabolic equivalent.

$P$ values are for overall differences by the $t$ test or ANOVA among males and females, age subgroups, ethnicities, income levels, alcohol consumption, smoking and exercise levels after adjusting for total energy intake: ${ }^{*} P<0.05$, ${ }^{* \star} P<0.01,{ }^{* \star *} P<0.001$.

$\dagger$ Antioxidant capacities of nutrients are expressed as $\mathrm{mg} \mathrm{VCE/d.}$

¥ Ratio of the median family income: poverty index. A PIR $\leq 1.30$ is required to be eligible for food assistance programs.

$\S$ Yes meant to consume twelve alcoholic beverages or more per year.

II Yes meant to have smoked cigarettes, cigars, pipes, or used chewing tobacco or snuff at least once during the past $30 \mathrm{~d}$.

" Exercise levels, expressed on the MET score, were calculated by combining the intensity level of the leisure-time activities reported, mean duration and frequency. 
Table 2. Dietary total antioxidant capacity (TAC) of US adults aged $19+$ years by food or food group consumption: National Health and Nutrition Examination Survey 2001-2

(Mean values with their standard errors)

\begin{tabular}{|c|c|c|c|c|c|c|c|c|c|}
\hline & \multicolumn{2}{|c|}{ Non-consumers* } & \multicolumn{2}{|c|}{ T1† } & \multicolumn{2}{|c|}{ T2† } & \multicolumn{2}{|c|}{ T3† } & \multirow[b]{2}{*}{$P \ddagger$} \\
\hline & Mean & SE & Mean & SE & Mean & SE & Mean & SE & \\
\hline \multicolumn{10}{|l|}{ Fruits and fruit juices $(\mathrm{g} / \mathrm{d})$} \\
\hline Range $(g / d)$ & \multirow{2}{*}{\multicolumn{2}{|c|}{$\begin{array}{c}0 \\
1485\end{array}$}} & \multirow{2}{*}{\multicolumn{2}{|c|}{$\begin{array}{c}\leq 124.5 \\
969\end{array}$}} & \multirow{2}{*}{\multicolumn{2}{|c|}{$\begin{array}{c}\leq 300 \cdot 81 \\
970\end{array}$}} & \multicolumn{2}{|c|}{$>300 \cdot 81$} & \\
\hline Subjects $(n)$ & & & & & & & & & \\
\hline TAC from total food intake (mg VCE/d) & 287.5 & $24 \cdot 8$ & 342.5 & $21 \cdot 2$ & $386 \cdot 0$ & $16 \cdot 1$ & $567 \cdot 2$ & $22 \cdot 7$ & 0.000 \\
\hline \multicolumn{10}{|l|}{ Vegetables and vegetable products (g/d) } \\
\hline Range (g/d) & \multirow{2}{*}{\multicolumn{2}{|c|}{$\begin{array}{c}0 \\
363\end{array}$}} & \multirow{2}{*}{\multicolumn{2}{|c|}{$\begin{array}{c}\leq 124.4 \\
1341\end{array}$}} & \multirow{2}{*}{\multicolumn{2}{|c|}{$\begin{array}{c}\leq 267.4 \\
1346\end{array}$}} & \multirow{2}{*}{\multicolumn{2}{|c|}{$\begin{array}{c}>267 \cdot 4 \\
1341\end{array}$}} & \\
\hline Subjects $(n)$ & & & & & & & & & \\
\hline TAC from total food intake (mg VCE/d) & $270 \cdot 8$ & 31.2 & 292.4 & $15 \cdot 8$ & $386 \cdot 2$ & $26 \cdot 3$ & $482 \cdot 9$ & 23.9 & 0.001 \\
\hline \multicolumn{10}{|l|}{ Bread and grain foods $(\mathrm{g} / \mathrm{d})$} \\
\hline Range $(g / d)$ & \multirow{2}{*}{\multicolumn{2}{|c|}{$\begin{array}{c}0 \\
87\end{array}$}} & \multirow{2}{*}{\multicolumn{2}{|c|}{$\begin{array}{l}\leq 592 \\
1433\end{array}$}} & \multicolumn{2}{|c|}{$\leq 1230$} & \multicolumn{2}{|c|}{$>1230$} & \\
\hline Subjects $(n)$ & & & & & & & & & \\
\hline TAC from total food intake (mg VCE/d) & 341.0 & $72 \cdot 3$ & 328.5 & $18 \cdot 9$ & 391.0 & $15 \cdot 6$ & $419 \cdot 2$ & $25 \cdot 5$ & 0.724 \\
\hline \multicolumn{10}{|l|}{ Beverages $\S(\mathrm{g} / \mathrm{d})$} \\
\hline Range $(\mathrm{g} / \mathrm{d})$ & \multirow{2}{*}{\multicolumn{2}{|c|}{$\begin{array}{c}0 \\
282\end{array}$}} & \multirow{2}{*}{\multicolumn{2}{|c|}{$\begin{array}{c}\leq 121.4 \\
1373\end{array}$}} & \multirow{2}{*}{\multicolumn{2}{|c|}{$\begin{array}{c}\leq 223.0 \\
1368\end{array}$}} & \multirow{2}{*}{\multicolumn{2}{|c|}{$\begin{array}{c}>223 \cdot 0 \\
1368\end{array}$}} & \\
\hline Subjects $(n)$ & & & & & & & & & \\
\hline TAC from total food intake (mg VCE/d) & $246 \cdot 7$ & $12 \cdot 1$ & $258 \cdot 0$ & $7 \cdot 3$ & 333.8 & $17 \cdot 3$ & $518 \cdot 7$ & $29 \cdot 2$ & $<0.001$ \\
\hline \multicolumn{10}{|l|}{ Wines $(g / d)$} \\
\hline Range $(\mathrm{g} / \mathrm{d})$ & \multirow{2}{*}{\multicolumn{2}{|c|}{$\begin{array}{c}0 \\
4048\end{array}$}} & \multirow{2}{*}{\multicolumn{2}{|c|}{$\begin{array}{c}\leq 33 \cdot 0 \\
115\end{array}$}} & \multirow{2}{*}{\multicolumn{2}{|c|}{$\begin{array}{c}\leq 206 \cdot 5 \\
117\end{array}$}} & \multirow{2}{*}{\multicolumn{2}{|c|}{$\begin{array}{c}>206 \cdot 5 \\
111\end{array}$}} & \\
\hline Subjects $(n)$ & & & & & & & & & \\
\hline TAC from total food intake (mg VCE/d) & 354.50 & $15 \cdot 0$ & $312 \cdot 5$ & 30.5 & $566 \cdot 0$ & $60 \cdot 6$ & $944 \cdot 0$ & $32 \cdot 4$ & 0.000 \\
\hline \multicolumn{10}{|l|}{ Teas $(\mathrm{g} / \mathrm{d})$} \\
\hline Range $(\mathrm{g} / \mathrm{d})$ & & & & & & & & & \\
\hline Subjects $(n)$ & & & & & & & & & \\
\hline TAC from total food intake (mg VCE/d) & $240 \cdot 3$ & $9 \cdot 8$ & $364 \cdot 3$ & 23.8 & $615 \cdot 3$ & 20 & $1345 \cdot 5$ & $52 \cdot 5$ & 0.000 \\
\hline
\end{tabular}

*All subjects who did not consume the food in $24 \mathrm{~h}$ dietary recalls were proposed as group 'non-consumers' and all consumers were divided into tertiles by the amount of consumption.

†T1, T2 and T3 refer to the first, second and third tertiles among the consumers of food or food group.

$\ddagger$ Adjusted for sex, age, ethnicity and total energy intake.

$\S$ Beverages include other drinks except wines, teas and fruit juices.

phytochemicals, such as flavonoids and PA, provided relatively high TAC (39 and 17\%, respectively), while those kinds of supplements which were expressed as the total amount of flavonoid and PA intakes regardless of the subcategories consumed accounted for so little $(<2 \%)$ and they were not included in the present study. The major sources of the phenolics in US diets were from fruits than from vegetables $^{(21)}$, black and green teas, red wines and cocoa $^{(42,43)}$. The significant contribution of flavonoids to TAC was similar to the previous findings that flavonoid consumption from diet was two times more than vitamin $C$ intake ${ }^{(10)}$. On the TAC database, flavonoids also have higher antioxidant capacities than other antioxidant vitamins ${ }^{(11)}$. Vitamin E and carotenoids took a very small part in TAC, which was attributed to their lower existence and their relatively lower antioxidant capacities ${ }^{(11)}$.

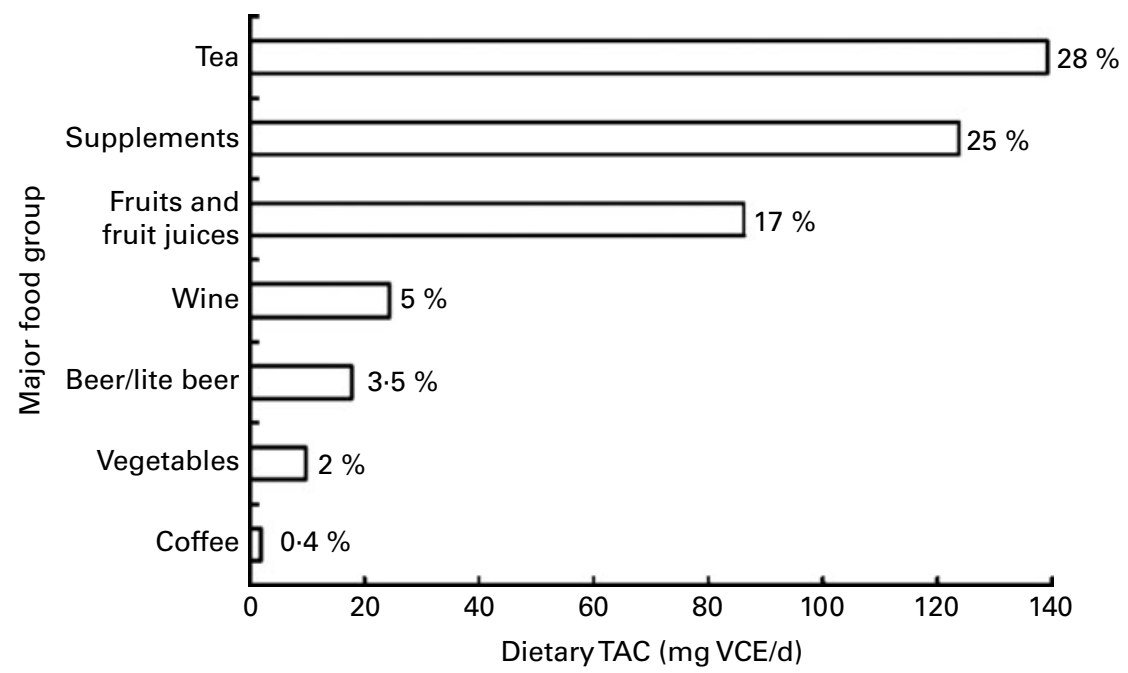

Fig. 1. Major sources of dietary total antioxidant capacity (TAC) in the US population aged $19+$ years: the National Health and Nutrition Examination Survey 2001-2, including the dietary TAC value of the major food groups and the percentage the food group contributes to the TAC. VCE, vitamin C equivalents. 
Table 3. Top major food items contributing to dietary total antioxidant capacity (TAC) in $19+$ years US adults

\begin{tabular}{|c|c|c|c|c|}
\hline Rank & Food groups & Dietary TAC (mg VCE/d) & $\%^{*}$ & Cum\% $†$ \\
\hline 1 & Teał & $139 \cdot 2$ & 27.6 & $27 \cdot 6$ \\
\hline 2 & Vitamin C supplement & $110 \cdot 8$ & $22 \cdot 0$ & $49 \cdot 6$ \\
\hline 3 & Orange juice§ & $27 \cdot 0$ & $5 \cdot 4$ & $55 \cdot 0$ \\
\hline 4 & Wine (table and dry) & 24.4 & 4.8 & $59 \cdot 8$ \\
\hline 5 & Beer\| & $17 \cdot 8$ & 3.5 & $63 \cdot 3$ \\
\hline 6 & Vitamin E supplement & $13 \cdot 0$ & 2.6 & 65.9 \\
\hline 7 & Banana (raw) & $7 \cdot 3$ & 1.5 & $67 \cdot 4$ \\
\hline 8 & Blueberries (raw) & 5.9 & $1 \cdot 2$ & $68 \cdot 6$ \\
\hline 9 & Orange (raw) & 5.4 & 1.1 & $69 \cdot 7$ \\
\hline 10 & Apple (raw) & $5 \cdot 0$ & 1.0 & $70 \cdot 7$ \\
\hline 11 & Fruit drink & 4.6 & 0.9 & $71 \cdot 6$ \\
\hline 12 & Strawberries (raw) & $3 \cdot 2$ & 0.6 & $72 \cdot 2$ \\
\hline 13 & Apple juice & $3 \cdot 1$ & 0.6 & $72 \cdot 8$ \\
\hline 14 & Cranberry juice drink with vitamin $\mathrm{C}$ added & $2 \cdot 8$ & 0.6 & 73.4 \\
\hline 15 & Tomato (raw) & $2 \cdot 7$ & 0.5 & 73.9 \\
\hline 16 & White potato** & 2.5 & 0.5 & 74.4 \\
\hline 17 & Grapefruit (raw) & $2 \cdot 3$ & 0.5 & 74.9 \\
\hline 18 & Plum (raw) & $2 \cdot 3$ & 0.5 & 75.4 \\
\hline 19 & Orange drink†† & $2 \cdot 1$ & 0.4 & $75 \cdot 8$ \\
\hline 20 & Coffee (made from ground and regular) & 1.9 & 0.4 & $76 \cdot 2$ \\
\hline 21 & Peach (raw) & 1.9 & 0.4 & $76 \cdot 6$ \\
\hline 22 & Cantaloupe (muskmelon, raw) & 1.8 & 0.4 & $77 \cdot 0$ \\
\hline 23 & Grapefruit juice (canned, bottled or in a carton, unsweetened) & 1.8 & 0.4 & $77 \cdot 4$ \\
\hline 24 & Chili con carne with beans & 1.8 & 0.4 & $77 \cdot 8$ \\
\hline 25 & Pear (raw) & 1.6 & 0.3 & $78 \cdot 1$ \\
\hline 26 & Onions (mature and raw) & 1.6 & 0.3 & 78.4 \\
\hline 27 & Broccoli (cooked, from fresh and fat not added in cooking) & 1.6 & 0.3 & $78 \cdot 7$ \\
\hline 28 & Lemonade & 1.6 & 0.3 & $79 \cdot 0$ \\
\hline 29 & Cherries (sweet, raw; Queen Anne, Bing) & 1.4 & 0.3 & $79 \cdot 3$ \\
\hline 30 & Grapes (raw, NS as to type) & 1.4 & 0.3 & $79 \cdot 6$ \\
\hline
\end{tabular}

VCE, vitamin C equivalents; Cum\%, cumulative percentage; NS, not specified.

* The percentage of the food item contributing to dietary TAC.

+ Cum $\%$ of the food items contributing to dietary TAC.

$\ddagger$ Includes tea, leaf, unsweetened; tea, leaf, pre-sweetened with sugar; tea, NS as to type, unsweetened; tea, NS as to type, pre-sweetened with sugar; tea, NS as to type, pre-sweetened, NS as to sweetener; tea, leaf, pre-sweetened with low-energy sweetener; tea, NS as to type, pre-sweetened with low-energy sweetener; tea, leaf, decaffeinated, unsweetened; tea, leaf, pre-sweetened, NS as to sweetener.

§Includes orange juice, canned, bottled or in a carton, unsweetened; orange juice, frozen, unsweetened (reconstituted with water).

|| Includes regular beer and lite beer.

I Includes fruit punch, fruit drink, or fruitade, with vitamin C added; fruit-flavoured drink, made from sweetened powdered mix (fortified with vitamin C); fruit juice drink, not fully specified.

** Includes white potato, French fries, from frozen, deep fried; white potato, chips.

†† Includes orange drink and orangeade with vitamin $\mathrm{C}$ added; orange breakfast drink.

TAC and individual antioxidant TAC from diet or supplements differed among sociodemographic subgroups. Energy-adjusted TAC of individual antioxidants increased with age, income and exercise levels except for carotenoids. This result was slightly different from the antioxidant intake estimation study ${ }^{(10)}$, in which flavonoids present no trend. The discrepancy was probably attributed to the fact that the low antioxidant capacity of carotenoids weakened their contribution to the whole diet, particularly in men, while the high antioxidant capacity of flavonoids strengthened their role in scavenging free radicals. One of the serious public concerns related to smoking is the lower consumption of vitamin C, vitamin $\mathrm{E}$ and carotenes by smokers ${ }^{(10)}$. The present study, however, did not find any differences of TAC intake between smokers and non-smokers, which may stem from the lower antioxidant capacities possessed by these three nutrients. Although many studies have proved that smoking causes smokers to be subject to higher oxidative stress than their counterpart, based on our studies, smoking people did not intend to consume more antioxidants to counteract the adverse damage from smoking, which, indeed, needs public and self concern to increase the corresponding consumption of the antioxidant-rich foods or supplements. Energy-adjusted TAC indicated the similar trend to the TAC of the individual antioxidants, that is, higher in men, older adults, Caucasians and those with higher income level and exercise level.

Consumption of fruits and fruit juices, vegetables and vegetable products, beverages, wines and teas was positively associated with dietary TAC (Table 2). Although the previous studies have reported that fruits and fruit juices, and vegetables have been identified as major sources of antioxidant vitamins $^{(44)}$, vitamins did not contribute the most to the TAC from food. Vitamin $\mathrm{C}$, the most abundant vitamin, was found to only account for less than $15 \%$ of antioxidant capacities in most fruits except for kiwi fruit and honeydew melon $^{(21)}$, whereas flavonoids were the predominant sources. Particularly, anthocyanins, with a high antioxidant potential among flavonoids, are abundant in many deep-coloured fruits and vegetables ${ }^{(45,46)}$ and in red wine ${ }^{(10,46)}$. Tea was demonstrated to be the major source of flavan-3-ols and flavonols, both of which accounted for over $90 \%$ of total flavonoids ${ }^{(47)}$. These findings suggested that the food items 
possessing high proportional flavonoids would contribute most to the dietary TAC.

We identified the major food sources of dietary TAC as tea, supplements, fruits and fruit juices and wines, while vegetables and vegetable products account for little antioxidant potential in the US diet. One previous study has implicated that only $21 \%$ of US adults drink tea daily, while fruits and fruit juices, were consumed by almost $80 \%$ of US adults ${ }^{(47)}$; the various abundances of flavonoids and vitamins prompt tea to have a stronger free-radical scavenging power. The prevalent consumption of supplements in US adults drives them to be a major contributor of TAC. Vegetables and vegetable products account for a tiny part of the TAC in the US diet, and potatoes, onions and broccoli were the top three dietary TAC contributors among different vegetables (Table 3). The findings about vegetable contribution were different from two previous TAC estimations based on FFQ, which found vegetables to be the main contributors to TAC intake in the Italian and Swedish population ${ }^{(48,49)}$. The comparison probably raised the public concern that food selection based on TAC may modify the lifestyles or health conditions in US populations.

To our best knowledge, the present study is the first to document the baseline dietary TAC levels in the free-living US populations on a large scale. It provides a general insight of the real 'quenching' power of antioxidants and broadens our horizon to assess the antioxidant functions. However, there are some limitations. First, the lack of some antioxidant intake data or antioxidant capacity values limits our investigation, such as Se, whose antioxidant capacity was difficult to measure. Second, the NHANES food consumption data were based on a $24 \mathrm{~h}$ DR that might not be accurately presenting the usual US diet. Third, our study focuses on the dietary data without considering the bioavailability or metabolism of the antioxidants. Dietary TAC is not an 'intrinsic' parameter for the human body; however, several studies have found that it was potentially related to some biomarkers of chronic diseases $^{(27,29)}$

The present study as a prerequisite for the future investigation of the association between the antioxidant status in humans and the risks of chronic diseases may advance the understanding of establishing the recommended dietary antioxidant intake in US populations for promoting the public health. Additionally, consumption of vegetables and fruits has been proved to decrease the risks of chronic diseases, though, whether antioxidants play the pivotal role warrants more investigation. Furthermore, the modulation of dietary TAC on plasma TAC or the role of antioxidant-rich diets on plasma antioxidant status is still debatable, which may be attributed to the homeostatic mechanisms of regulation, the various bioavailability of different antioxidants and the methods used for measuring biological antioxidant status or TAC. Importantly, on the journey of researching the dietary or physiological antioxidant status or their potential power for fighting against oxidative stress and therefore chronic diseases, considering antioxidants as a whole group, that is, as TAC, instead of individual nutrients, is a better direction.

\section{Acknowledgements}

The present study was fully funded by the Beginning Grant-inAid no. 0865092E from the American Heart Association. The authors bear no conflict of interest regarding the manuscript submitted to the British Journal of Nutrition. The article was partially presented at the 2010 Experimental Biology Meeting, Anaheim, CA, in April 2010. O. K. C. and W. O. S. designed the study. D. O.-K. prepared the preliminary studies. S.-J. C. and C. E. C. performed the statistical analyses. M. Y. analysed and interpreted the TAC data. S. I. K. and W. O. S. provided technical support and advice as members of the project steering group. All authors were involved in the data interpretation and manuscript preparation.

\section{References}

1. Hertog MG, Feskens EJ, Hollman PC, et al. (1993) Dietary antioxidant flavonoids and risk of coronary heart disease: the Zutphen Elderly Study. Lancet 342, 1007-1011.

2. Hertog MG, Kromhout D, Aravanis C, et al. (1995) Flavonoid intake and long-term risk of coronary heart disease and cancer in the seven countries study. Arch Intern Med 155, $381-386$.

3. Hertog MG, Sweetnam PM, Fehily AM, et al. (1997) Antioxidant flavonols and ischemic heart disease in a Welsh population of men: the Caerphilly Study. Am J Clin Nutr $\mathbf{6 5}$, 1489-1494.

4. Doll R (1990) An overview of the epidemiological evidence linking diet and cancer. Proc Nutr Soc 49, 119-131.

5. Hertog ML, Sweetnam P, Fehily A, et al. (1997) Potentially Anticarcinogenic Secondary Metabolites from Fruit and Vegetables. Oxford: Clarendon Press.

6. Serafini M \& Del Rio D (2004) Understanding the association between dietary antioxidants, redox status and disease: is the total antioxidant capacity the right tool? Redox Rep 9, $145-152$.

7. Ghiselli A, Serafini M, Natella F, et al. (2000) Total antioxidant capacity as a tool to assess redox status: critical view and experimental data. Free Radic Biol Med 29, 1106-1114.

8. Chun OK, Chung S-J \& Song WO (2007) Estimated dietary flavonoid intakes and major food sources of U.S. adults. J Nutr 137, 1244-1252.

9. Chun O, Chung S-J \& Song WO (2009) Estimated intakes of proanthocyanidin in the US population. Experimental Biology Meeting 2009, April 20, 2009, New Orleans, LA: FASEB.

10. Chun OK, Floegel A, Chung S-J, et al. (2010) Estimation of antioxidant intakes from diet and supplements in U.S. adults. J Nutr 140, 317-324.

11. Floegel A, Kim D-O, Chung S-J, et al. (2010) Development and validation of an algorithm to establish a total antioxidant capacity database of the US diet. Int J Food Sci Nutr 61, $600-623$.

12. National Center for Health Statistics (2004) National Health and Nutrition Examination Survey, 2001-2002 Data Files. Hyattsville, MD: Center for Disease Control. http://www. cdc.gov/nchs/data/nhanes/nhanes_01_02/136_b_doc.pdf (accessed May 2004).

13. van Herpen-Broekmans WMR, Klopping-Ketelaars IAA, Bots ML, et al. (2004) Serum carotenoids and vitamins in relation to markers of endothelial function and inflammation. Eur J Epidemiol 19, 915-921. 
14. Botman S, Moore T, Moriarity C, et al. (2000) Design and estimation for the national health interview survey, 1995-2004. Vital Health Stat 2 130, 1-31.

15. Agricultural Research Service \& US Department of Agriculture (2003) Database for the Flavonoid Content of Selected Foods. Beltsville, MD: Agricultural Research Service. http:// www.nal.usda.gov/fnic/foodcomp/Data/Flav/flav.pdf (accessed March 2003)

16. Agricultural Research Service \& US Department of Agriculture (2002) USDA-Iowa State University Database on the Isoflavone Content of Foods, Release 1.3. Beltsville, MD: Agricultural Research Service. http://www.nal.usda.gov/fnic/ foodcomp/Data/isoflav/isoflav.html (accessed 2002).

17. Agricultural Research Service \& US Department of Agriculture (2004) Database for the Proanthocyanidin Content of Selected Foods. Beltsville, MD: Agricultural Research Service. http://www.nal.usda.gov/fnic/foodcomp/Data/PA/ PA.html (accessed 2004).

18. National Center for Health Statistics (2002) National Health and Nutrition Examination Survey, 1999-2000 Data Files. Hyattsville, MD: Centers for Disease Control. http://www. cdc.gov/nchs/nhanes/nhanes1999-2000/nhanes99_00.htm (accessed 2002).

19. Kim D-O, Chun OK, Kim YJ, et al. (2003) Quantification of polyphenolics and their antioxidant capacity in fresh plums. J Agric Food Chem 51, 6509-6515.

20. Kim D-O, Lee KW, Lee HJ, et al. (2002) Vitamin C equivalent antioxidant capacity (VCEAC) of phenolic phytochemicals. J Agric Food Chem 50, 3713-3717.

21. Chun OK, Kim D-O, Smith N, et al. (2005) Daily consumption of phenolics and total antioxidnat capacity from fruits and vegetables in the American diet. $J$ Sci Food Agric 85, 1715-1724.

22. Gey KF (1990) The antioxidant hypothesis of cardiovascular disease: epidemiology and mechanisms. Biochem Soc Trans 18, $1041-1045$.

23. Lonn E, Bosch J, Yusuf S, et al. (2005) Effects of long-term vitamin $\mathrm{E}$ supplementation on cardiovascular events and cancer: a randomized controlled trial. JAMA 293, 1338-1347.

24. Dalgård C, Nielsen F, Morrow J, et al. (2009) Supplementation with orange and blackcurrant juice, but not vitamin $\mathrm{E}$, improves inflammatory markers in patients with peripheral arterial disease. Br J Nutr 101, 263-269.

25. Eidelman RS, Hollar D, Hebert PR, et al. (2004) Randomized trials of vitamin $\mathrm{E}$ in the treatment and prevention of cardiovascular disease. Arch Intern Med 164, 1552-1556.

26. Markovits N, Ben AA \& Levy Y (2009) The effect of tomatoderived lycopene on low carotenoids and enhanced systemic inflammation and oxidation in severe obesity. Is $r$ Med Assoc J 11, 598-602.

27. Brighenti F, Valtuena S, Pellegrini N, et al. (2005) Total antioxidant capacity of the diet is inversely and independently related to plasma concentration of high-sensitivity C-reactive protein in adult Italian subjects. Br J Nutr 93, 619-625.

28. Detopoulou P, Panagiotakos DB, Chrysohoou C, et al. (2010) Dietary antioxidant capacity and concentration of adiponectin in apparently healthy adults: the ATTICA Study. Eur J Clin Nutr 64, 161-168.

29. Puchau B, Zulet MA, de Echavarri AG, et al. (2009) Dietary total antioxidant capacity is negatively associated with some metabolic syndrome features in healthy young adults. Nutrition 26, 534-541.

30. Pellegrini N, Salvatore S, Valtuena S, et al. (2007) Development and validation of a food frequency questionnaire for the assessment of dietary total antioxidant capacity. $J$ Nutr 137, 93-98.
31. Miller N, Rice-Evans C, Davies M, et al. (1993) A novel method for measuring antioxidant capacity and its application to monitoring the antioxidant status in premature neonates. Clin Sci (London) 84, 407-412.

32. Cao G, Alessio HM \& Cutler RG (1993) Oxygen-radical absorbance capacity assay for antioxidants. Free Radic Biol Med 14, 303-311.

33. Ghiselli A, Serafini M, Maiani G, et al. (1995) A fluorescencebased method for measuring total plasma antioxidant capability. Free Radic Biol Med 18, 29-36.

34. Benzie IF \& Strain JJ (1996) The ferric reducing ability of plasma (FRAP) as a measure of "antioxidant power": the FRAP assay. Anal Biochem 239, 70-76.

35. Brand-Williams W, Cuvelier ME \& Berset C (1995) Use of a free radical method to evaluate antioxidant activity. Lebensm Wiss Technol 28, 25-30.

36. Fernandez-Panchon MS, Villano D, Troncoso AM, et al. (2008) Antioxidant activity of phenolic compounds: from in vitro results to in vivo evidence. Crit Rev Food Sci Nutr 48, 649-671.

37. Prior RL, Wu X \& Schaich K (2005) Standardized methods for the determination of antioxidant capacity and phenolics in foods and dietary supplements. J Agric Food Chem 53, 4290-4302.

38. Kim D-O \& Lee CY (2004) Comprehensive study on vitamin C equivalent antioxidant capacity (VCEAC) of various polyphenolics in scavenging a free radical and its structural relationship. Crit Rev Food Sci Nutr 44, 253-273.

39. Valtuena S, Pellegrini N, Franzini L, et al. (2008) Food selection based on total antioxidant capacity can modify antioxidant intake, systemic inflammation, and liver function without altering markers of oxidative stress. Am J Clin Nutr 87, 1290-1297.

40. Rautiainen S, Serafini M, Morgenstern R, et al. (2008) The validity and reproducibility of food-frequency questionnaire-based total antioxidant capacity estimates in Swedish women. Am J Clin Nutr 87, 1247-1253.

41. USDA (2007) Database for the Oxygen Radical Absorbance Capacity (ORAC) of Selected Foods. Beltsville, MD: Nutrient Data Laboratory, Beltsville Human Nutrition Research Center.

42. Lee KW, Kim YJ, Lee HJ, et al. (2003) Cocoa has more phenolic phytochemicals and a higher antioxidant capacity than teas and red wine. J Agric Food Chem 51, 7292-7295.

43. Cao G, Sofic E \& Prior RL (1996) Antioxidant capacity of tea and common vegetables. J Agric Food Chem 44, 3426-3431.

44. Hampl JS, Taylor CA \& Johnston CS (1999) Intakes of vitamin $\mathrm{C}$, vegetables and fruits: which schoolchildren are at risk? J Am Coll Nutr 18, 582-590.

45. Yang M, Koo SI, Song WO, et al. (2011) Food matrix affecting anthocyanin bioavailability: review. Curr Med Chem 18, 291-300.

46. Proteggente AR, Pannala AS, Paganga G, et al. (2002) The antioxidant activity of regularly consumed fruit and vegetables reflects their phenolic and vitamin C composition. Free Radic Res 36, 217-233.

47. Song WO \& Chun OK (2008) Tea is the major source of flavan-3-ol and flavonol in the U.S. diet. J Nutr $\mathbf{1 3 8}$ 1543S-1547S.

48. Rautiainen S, Serafini M, Morgenstern R, et al. (2008) The validity and reproducibility of food-frequency questionnaire-based total antioxidant capacity estimates in Swedish women. Am J Clin Nutr 87, 1247-1253.

49. Pellegrini N, Salvatore S, Valtuena S, et al. (2007) Development and validation of a food frequency questionnaire for the assessment of dietary total antioxidant capacity. $J$ Nutr 137, 93-98. 